\title{
ENTREVISTA A MARTA ARUMÍ RIBAS
}

\author{
Carlos Henrique Rodrigues 1 \\ ${ }^{1}$ Universidade Federal de Santa Catarina, Florianópolis, SC, Brasil \\ Macarena Dehnhardt Amengual 2 \\ ${ }_{2}^{2}$ Universitat Autònoma de Barcelona, Barcelona, Bellaterra, Espanha
}

Marta Arumí Ribas es Doctora en Traducción e Interpretación por la Universitat Pompeu Fabra, donde obtuvo el premio extraordinario de doctorado. También es Licenciada en Traducción e Interpretación por la Universitat Autònoma de Barcelona (UAB). Cursó el Máster en Interpretación de Conferencias por la Universidad de La Laguna y el Máster en Formación de Formadores en Interpretación de Conferencias de la Escuela de Traducción e Interpretación de la Universidad de Ginebra. Actualmente, es profesora agregada del departamento de Traducción, Interpretación y Estudios de Asia Oriental de la UAB, en donde imparte clases a nivel de grado y postgrado. Además, es la coordinadora del Máster Universitario en Interpretación de Conferencias en esta misma universidad. Su interés investigador se centra en la didáctica de la interpretación de conferencias y en la interpretación en los servicios públicos, temas sobre los cuales ha escrito artículos especializados en revistas nacionales e internacionales y ha dirigido varias tesis doctorales. Asimismo, es investigadora principal del grupo MIRAS (por sus siglas en catalán: Mediació i Interpretació: Recerca en l'Àmbit Social [Mediación e interpretación: investigación en el ámbito social]). Este grupo se dedica al estudio de la Interpretación en los Servicios públicos y de la mediación lingüística y cultural en diversos ámbitos, como lo son el judicial, el educativo y el sanitario. Desde su 
creación, en el año 2009, la Dra. Arumí ha dirigido variados proyectos de investigación financiados por entidades gubernamentales.

Cadernos de Tradução (CT): ¿Cómo se vinculó usted a la traducción y a la interpretación en los servicios públicos en particular?

Marta Arumí Ribas (MAR): Mi vínculo con la traducción se remonta al año 1992, cuando inicié la Licenciatura en Traducción e Interpretación en la Universitat Autònoma de Barcelona. Mi interés por la interpretación de conferencias, me llevó a graduarme, en el año 1997, en el Máster de Interpretación de Conferencias de la Universidad de La Laguna. Tras ejercer como intérprete profesional, empezó a interesarme la investigación y la docencia de la interpretación. Entre los años 2000 y 2007, ejercí como profesora en la Universitat Pompeu Fabra, en la que a su vez me doctoré en el año 2006. Durante ese periodo, mis intereses investigadores se focalizaron mayormente en la interpretación de conferencias, concretamente en la didáctica de la modalidad consecutiva. El año 2007 me incorporé al Departamento de Traducción e Interpretación y Estudios de Asia Oriental de la UAB. En esos años, Cataluña, a semejanza de otros países, se estaba convirtiendo en tierra de acogida de inmigrantes y la administración pública catalana empezaba a demandar traductores e intérpretes que pudieran solucionar los problemas de comunicación entre la población recién llegada y los funcionarios de la administración. La necesidad social empezaba a reclamar una respuesta de la universidad, tanto a nivel de formación como de investigación. Fue así como en el año 2009, y fruto de la iniciativa de un grupo de investigadoras del Departamento de Traducción e Interpretación y Estudios de Asia Oriental de la UAB, nació el grupo de investigación MI$\mathrm{RAS}^{1}$, del que soy su investigadora principal desde sus inicios. El objetivo del grupo es el estudio de la comunicación entre comuni-

${ }^{1}$ Página web del grupo MIRAS: http://grupsderecerca.uab.cat/miras/

Cad. Trad., Florianópolis, v. 41, no 2 p. 457-478, mai-ago, 2021. 
dades lingüísticas diversas y del perfil profesional del intérprete en los servicios públicos, que hace que esa comunicación no solo resulte posible, sino también efectiva.

(CT): En sus palabras, ¿defina qué es la interpretación a diferencia de otros procesos de traducción?

$(M A R)$ : La interpretación es la actividad traductora que permite la comunicación oral, o a través de signos, entre dos o varias personas que no comparten el mismo código lingüístico ni cultural. El intérprete es un profesional que realiza un proceso cognitivo muy complejo y con una vertiente humana y de interacción que influyen directamente en su práctica. La función principal del intérprete es la de transmitir las ideas de un idioma a otro, sin adaptar ni modificar el mensaje. Pero más allá de ser vehículo de comunicación entre lenguas y culturas diversas, el intérprete también es un vehículo de cohesión e integración social. Sin garantías de comunicación, nos hallamos ante un peligro de vulneración de los derechos fundamentales. Si bien la labor del intérprete de conferencias goza de reconocimiento y su función es clara, todavía hay numerosos malentendidos entorno a la figura y a la labor del intérprete en los servicios públicos. La administración suele pecar de indefinición de funciones laborales, sin marcar claramente las diferencias existentes entre traducción escrita e interpretación. Traducir e interpretar son dos actividades y habitualmente dos profesiones diferentes. Quien traduce trabaja con la lengua escrita y quien interpreta lo hace con la lengua oral o de signos. Además, en más ocasiones de las deseadas, se considera a los intérpretes en los servicios públicos como auxiliares de otros profesionales, se desconoce la especialización que implica su labor o bien se suele caer en la creencia errónea de que basta con dominar una o más lenguas extranjeras para poder interpretar. Nada más lejos de la realidad. Hace falta mucho más. 
(CT): ¿Cuáles son los ámbitos más habituales de la interpretación?

(MAR): Si pensamos en la interpretación de conferencias, concretamente en la modalidad de simultánea, los ámbitos habituales son los foros internacionales, así como los congresos y conferencias especializadas. En el caso de la consecutiva, se suele usar más en entrevistas o ruedas de prensa. En el caso de la Interpretación en los Servicios Públicos (ISP), la división tradicional son los ámbitos educativo, sanitario y de atención al ciudadano y el ámbito judicial, que puede incluir también el contexto policial.

(CT): ¿Hay alguno ámbito, en su opinión, en el que la interpretación es más compleja? ¿Por qué?

(MAR): La interpretación es una actividad compleja de por sí. Yo no diría que existe un ámbito más complejo que otro sino situaciones de interpretación más complicadas. Por ejemplo, en interpretación de conferencias, es más complicado interpretar a un orador que está leyendo un discurso a velocidad excesiva que a un orador que transmite espontáneamente sus ideas o que simplemente parte de un guion para construir su discurso. Es cierto que existen los campos de especialización, como la medicina, por ejemplo, para los que el intérprete debe estudiar, prepararse y documentarse exhaustivamente antes de la interpretación. En ISP, una de las principales dificultades radica en que el intérprete mantenga su neutralidad e imparcialidad. Asimismo, el intérprete en los servicios públicos debe estar preparado para interpretar registros y estilos muy diversos (desde el registro formal a vulgarismos e insultos), y lo debe hacer con precisión tanto si el discurso es difícil por los tecnicismos y la formalidad como si lo es porque se trata de un discurso desestructurado y difícil de seguir. También el intérprete en los servicios públicos tiene que enfrentarse a escollos a la hora de prepararse, ya sea por la cuestión de la confidencialidad mal entendida, por la escasa profesionalización 
o por el desconocimiento de quien contrata los servicios. Otra de las dificultades importantes de la ISP la hallamos en la gestión de las emociones, ya que una característica distintiva es el elevado componente emocional de los encuentros en los que el intérprete actúa. También en la ISP existen campos de especialización, como es el caso de la interpretación judicial.

(CT): Si echa la vista atrás, ¿cómo ve el pasado y la evolución profesional de los intérpretes de conferencia y comunitarios en Europa?

$(M A R)$ : En el siglo XXI, los estudios de interpretación han conseguido consolidar sus largas aspiraciones, tanto en relación con su carácter interdisciplinar como en el hecho de convertirse en una disciplina diferenciada, por su grado de especialización y por su gran número de publicaciones. Además, en la última década, también se ha consolidado la investigación en nuevas modalidades de interpretación. En la interpretación de conferencias, la profesión de intérprete hoy en día ha evolucionado respecto a los intérpretes de mediados del siglo XX, cuando tuvo lugar la gran eclosión de escuelas europeas de interpretación, y también en relación al año 1986, cuando España ingresó en la Unión Europea. Las nuevas tecnologías han flexibilizado la profesión y se han diversificado las formas más convencionales de ejercerla. Estas transformaciones han dado lugar a un perfil profesional pluridimensional capaz de dar respuesta a las nuevas necesidades del mercado laboral. La interpretación consecutiva, por la lentitud que introduce en la comunicación, ha venido perdiendo terreno ante la interpretación simultánea, y ésta se va usando en nuevos entornos, como en las videoconferencias o en la interpretación telefónica. Asimismo, cada vez hay más intérpretes de conferencia con las combinaciones lingüísticas más tradicionales y, en cambio, se requieren más expertos lingüísticos que dominen lenguas hasta ahora menos conocidas. En cuanto a la Interpretación en los Servicios Públicos, los avances en 
España se encuentran lejos de los de otros países con mayor tradición de recepción de inmigración. En España, la falta de formación, y sobre todo de una acreditación específica para intérpretes en los servicios públicos, supone una falta de regulación del ámbito que normalmente tiene una repercusión directa en la calidad de las interpretaciones. En el ámbito judicial, la directiva 2010/64/UE del Parlamento Europeo y del Consejo de 20 de octubre de 2010 relativa al derecho a interpretación y a traducción en los procesos penales, ha ofrecido a los países miembros de la UE la posibilidad de poner en marcha mecanismos para garantizar la calidad de la interpretación y de la traducción en los procesos penales a fin de que se pueda garantizar el derecho de defensa y la salvaguarda de la equidad del proceso. No obstante, en más ocasiones de las deseadas, nos hallamos todavía ante un intérprete sin formación, que se excede en sus funciones y que demuestra carencia de un marco ético que le permita regular su práctica. Desde la universidad, a través de la oferta docente y de la investigación, se ha otorgado a la ISP el valor que se merece. También las asociaciones profesionales han velado por la creación de pautas éticas y por la denuncia de malas prácticas. Por su parte, los profesionales que trabajan en su día a día con intérpretes han empezado a cuestionarse que las soluciones ad hoc que se suelen ofrecer no garantizan la buena provisión de los servicios. Pero, a pesar de que la investigación ha puesto de manifiesto que en España y en Cataluña existe un sistema que no funciona, la administración sigue mirando hacia otra parte y priorizando la facilidad en la gestión de los servicios y el lucro de ciertas empresas por encima de la vulneración constante de los derechos que suponen las malas prácticas de interpretación.

(CT): ¿En qué consiste la competencia del intérprete de los servicios públicos?

$(M A R):$ El perfil del intérprete en nuestras sociedades contemporáneas ya no es el que era veinte años atrás. Nos movemos hacia una 
figura más polivalente, más pluridimensional, capaz de adaptarse a modalidades y contextos diversos y de aportar, como valor añadido, algo más que el mero trasvase de términos o de ideas, para convertirse en un auténtico mediador, lingüístico y también cultural. El intérprete necesita un elevado dominio de los dos idiomas presentes en la conversación; conocimiento de las culturas de los interlocutores; conocimiento de las técnicas y las destrezas de la interpretación; capacidad de análisis y síntesis; conocimiento especializado sobre la temática del encuentro comunicativo; comportamiento acorde con el código ético que rige el ejercicio profesional $\mathrm{y}$ habilidades emocionales para hacer frente a situaciones difíciles. Pero, ante todo, es muy importante que el intérprete desarrolle su competencia estratégica, su capacidad de toma de decisiones rápida y de autoevaluación.

(CT): ¿Cuál es la formación ideal del intérprete? ¿Qué valor ha tenido y tiene la Universidad, desde su punto de vista, en esa formación?

$(M A R)$ : La enseñanza de la interpretación de conferencias es casi paralela en el tiempo a la creación de la profesión, que se considera que nace en los Juicios de Nuremberg, en los cuales se empieza a trabajar en la modalidad simultánea. En las décadas de 1940 y 1950, después de la Segunda Guerra Mundial, las grandes escuelas de intérpretes se establecieron alrededor de las principales organizaciones internacionales, que solían ser el destino natural de los intérpretes que se formaban. Los primeros programas habían empezado antes del final de la Segunda Guerra Mundial (Moscú, Heidelberg, Ginebra y Viena). Otros programas empezaron después del 1945 con el apoyo de las fuerzas aliadas (Innsbruck, Graz, Mainz/Germersheim, Saarbrücken, Paris y Washington) y, por último, en 1957 se crearon dos programas en Paris. En España, el primer centro de formación de traductores e intérpretes nació en la Universitat Autònoma de Barcelona en 1972 con la 
creación de la Escuela Universitaria de Traductores e Intérpretes (EUTI). El ejercicio de la interpretación requiere por parte del estudiante un dominio excepcional de su lengua materna, así como unos conocimientos lingüísticos y culturales muy amplios en un mínimo de dos idiomas extranjeros, que han de poder activarse de forma instantánea, sin poder revisar y sin poder recurrir a las fuentes de documentación habituales en la práctica de la traducción escrita. En la interpretación en los servicios públicos, en la que el intérprete trabaja generalmente en la modalidad de enlace, es decir, alterna dos códigos lingüísticos, el intérprete debe dominar al mismo nivel activo ambas lenguas con las que trabaja para poder prestar un servicio de calidad. A mi entender, la universidad juega un papel determinante en la formación de intérpretes de conferencia, al tratarse de una formación altamente especializada que requiere muchas horas de práctica y acompañamiento académico y profesional. Con los años, las universidades han creado equipos de profesores de interpretación en los que conviven profesores con perfiles más académicos e investigadores con profesionales del ámbito, que aportan al aula su experiencia diaria en la cabina. Estos equipos docentes mixtos son fundamentales para la formación en una disciplina muy técnica, que se enseña y se aprende a través de la práctica acompañada. Si bien los retos en la formación de intérpretes para los servicios públicos son otros, también el rol de la universidad es fundamental. Uno de los principales escollos de la formación en ISP radica en la multiplicidad de lenguas y combinaciones en las que hay que formar (chino, árabe, urdu, wolof, rumano, etc.) y que no son las combinaciones tradicionales en las que se suele formar en las facultades de traducción e interpretación en Europa. Asimismo, no suele existir profesorado formado que pueda impartir docencia y evaluar estas combinaciones. En algunos casos, se ha optado por ofrecer formaciones intralingüísticas, pero es una cuestión sobre la que cabe seguir reflexionando. En el ámbito de la ISP, la intervención de la universidad es clave para seguir avanzando hacia la profesionalización. No basta solo con la implicación de la universidad, pero es un actor importante para 
avanzar hacia la implantación de pruebas evaluables, objetivas y fiables, así como de una formación especializada.

(CT): Con la globalización de la sociedad ¿Qué habilidades específicas van a necesitar los futuros intérpretes?

$(M A R)$ : Por novedosos que sean los cambios, no hay que olvidar los fundamentos teóricos que garantizan la calidad de la enseñanza y el domino de las técnicas básicas de la interpretación $\mathrm{u}$ la consecutiva, la simultánea y la traducción a la vista $\square$ en las que se apoyan todas las modalidades interpretativas y que permiten al intérprete su adaptación al perfil y a las demandas de los mercados de trabajo. Lo que sí que es cierto es que, cada vez más, los profesores y los estudiantes deberán familiarizarse con nuevas herramientas tecnológicas como tabletas, ordenadores y teléfonos móviles y con nuevos espacios virtuales. Además de formar en las particularidades de la interpretación en nuevos espacios como la interpretación remota, en hubs, o incluso los intérpretes que interpretan desde casa, es muy importante reflexionar con los futuros intérpretes sobre la protección que hay que defender para poder seguir ofreciendo un trabajo de calidad y con garantías de precisión. Aquí podríamos pensar en cuestiones como en quién recae la responsabilidad por los posibles problemas técnicos, la inclusión de cláusulas abusivas en los contratos, etc. Tampoco podemos perder de vista que están apareciendo otros campos profesionales en los cuales pueden encontrar su espacio los profesionales formados en interpretación. El caso más paradigmático lo hallamos en el campo de la traducción audiovisual, con el subtitulado basado en el reconocimiento de voz, conocido también como respeaking, y que parece irse consolidando como el método por el que están optando muchas cadenas de televisión para proporcionar subtítulos en directo para la población sorda. 
(CT): No queremos acabar la entrevista sin hablar de sus investigaciones, pues la investigación es importante siempre para el desarrollo personal y profesional. Cuéntenos cómo se ha desarrollado la investigación en su carrera profesional y cómo surgió el grupo de investigación MIRAS.

$(M A R)$ : En mi caso, la investigación es la que ha alimentado siempre mi práctica. En mi primera etapa investigadora, en la que realicé mi tesis doctoral, me centré en la didáctica de la interpretación. Mi interés eran los procesos de enseñanza y aprendizaje de la interpretación consecutiva, sobre todo relacionados con los procesos metacognitivos de una aprendizaje altamente especializado y complejo. Desde el año 2007, me he centrado más en la investigación preocupada por las cuestiones sociales y con una aplicación clara a la sociedad. La investigación que incide en el cambio. La investigación que es justa socialmente, y que analiza los problemas que nos afectan directamente. Como he mencionado anteriormente, en el año 2009 creamos el grupo de investigación MIRAS, que integra investigadoras de la UAB, así como investigadoras de otras universidades nacionales e internacionales y colaboradoras vinculadas profesionalmente a la ISP y a la mediación intercultural. En los últimos años, y gracias en buena parte al trabajo pionero del grupo en proyectos de investigación competitivos, la ISP se ha convertido en una actividad reconocida tanto a nivel estatal como autonómico, y en una de las líneas de investigación con más crecimiento e impacto dentro de los estudios de traducción e interpretación. El carácter internacional e interdisciplinar del grupo ha propiciado una investigación innovadora y de calidad, vertebrada en torno a los ámbitos de actuación tradicionales dentro de la ISP. El grupo MIRAS ha realizado hasta el momento una investigación muy consistente, que nos ha permitido conocer muy bien la práctica de la ISP en Cataluña, las competencias del intérprete, las formas de trabajo, las modalidades y las necesidades de los intérpretes sociales en la actualidad. Asimismo, los resultados de la investigación nos han permitido materializar propuestas formativas y proponer 
un sistema de acreditación adecuado a nuestro contexto. No quisiera acabar esta entrevista sin mencionar el resultado del último proyecto $^{2}$ del grupo. Gracias a la confianza y a la financiación de la Dirección General de Interpretación de Conferencias de la Comisión Europea, se ha desarrollado un material audiovisual de libre acceso que consta de 7 videos destinados a la mejora del trabajo y la formación de los intérpretes en los servicios públicos en el contexto europeo. Estos videos, cada uno dedicado a un ámbito y a una especificidad, recogen los criterios que deberían aplicarse a la formación en ISP y son fruto del resultado de la reflexión y la investigación de los últimos 10 años.

${ }^{2}$ Más información en el siguiente enlace: http://pagines.uab.cat/expandingpsi/

Carlos Henrique Rodrigues. E-mail: carlos.rodrigues@ufsc.br. ORCID: http:// orcid.org/0000-0002-5726-1485.

Macarena Dehnhardt Amengual. E-mail: macarena.dehnhardt@e-campus.uab. cat. ORCID: 